\title{
The Evolution-Dominated Hydrodynamic Model and the Pseudorapidity Distributions in High Energy Physics
}

\author{
Z. J. Jiang, H. L. Zhang, J. Wang, and K. Ma \\ College of Science, University of Shanghai for Science and Technology, Shanghai 200093, China \\ Correspondence should be addressed to Z. J. Jiang; jzj265@163.com
}

Received 22 January 2014; Accepted 2 March 2014; Published 3 April 2014

Academic Editor: Fu-Hu Liu

Copyright ( 2014 Z. J. Jiang et al. This is an open access article distributed under the Creative Commons Attribution License, which permits unrestricted use, distribution, and reproduction in any medium, provided the original work is properly cited. The publication of this article was funded by SCOAP S $^{3}$

\begin{abstract}
By taking into account the effects of leading particles, we discuss the pseudorapidity distributions of the charged particles produced in high energy heavy ion collisions in the context of evolution-dominated hydrodynamic model. The leading particles are supposed to have a Gaussian rapidity distribution normalized to the number of participants. A comparison is made between the theoretical results and the experimental measurements performed by BRAHMS and PHOBOS Collaboration at BNL-RHIC in Au-Au and $\mathrm{Cu}-\mathrm{Cu}$ collisions at $\sqrt{s_{\mathrm{NN}}}=200 \mathrm{GeV}$ and by ALICE Collaboration at CERN-LHC in Pb-Pb collisions at $\sqrt{s_{\mathrm{NN}}}=2.76 \mathrm{TeV}$.
\end{abstract}

\section{Introduction}

Along with the successful description of elliptic flow and multiplicity production in heavy ion collisions [1-4], relativistic hydrodynamics has now been widely accepted as one of the most important tools for understanding the spacetime evolution of the matter created in collisions [5-11]. With the specified initial conditions, the equation of state, and the freeze-out conditions, the motion of fluid relies only on the local energy-momentum conservation and the assumption of local thermal equilibrium. From this point of view, hydrodynamics is simple and powerful. However, on the other hand, the initial conditions, the equation of state, and the freeze-out conditions of fluid are not well known. Worse still is that the partial differential equations of relativistic hydrodynamics are highly nonlinear and coupled. It is a very hard thing to solve them analytically. From this point of view, hydrodynamics is tremendously complicated. This is the reason why the progress in finding exact hydrodynamic solutions is not going well. Up till now, most of this work is only limited in $1+1$ dimensional flows for the perfect fluid with the simple equation of state [12-23]. The $3+1$ dimensional hydrodynamics is less developed, and no general exact solutions are known so far.

In the present paper, by using the evolution-dominated hydrodynamics [12] and taking into account the contribution from leading particles, we will discuss the pseudorapidity distributions of the charged particles produced in heavy ion collisions. We will first give a brief introduction to the evolution-dominated hydrodynamics in Section 2. The obtained solutions are then used in Section 3 to formulate the rapidity distributions of charged particles produced in heavy ion collisions. Then, in Section 4 , a comparison is made between the theoretical results and experiment measurements carried out by BRAHMS and PHOBOS Collaboration at BNL-RHIC in Au-Au and $\mathrm{Cu}-\mathrm{Cu}$ collisions at $\sqrt{s_{\mathrm{NN}}}=$ $200 \mathrm{GeV}$ [24-27] and by ALICE Collaboration at CERN-LHC in $\mathrm{Pb}-\mathrm{Pb}$ collisions at $\sqrt{s_{\mathrm{NN}}}=2.76 \mathrm{TeV}$ [28]. The last section is traditionally about conclusions.

\section{A Brief Introduction to Evolution-Dominated Hydrodynamics}

Here, for the purpose of completion and applications, we will give a brief introduction to the evolution-dominated hydrodynamics [12].

The motion of a perfect fluid obeys the equation

$$
\frac{\partial T^{\mu v}}{\partial x^{\mu}}=0
$$


where $x^{\mu}=\left(x^{0}, x^{1}, x^{2}, x^{3}\right)=(t, z, x, y)$ is the 4 -vector of space-time and

$$
T^{\mu v}=(\varepsilon+p) u^{\mu} u^{v}-p g^{\mu v}
$$

is the energy-momentum tensor, $g^{\mu \nu}=\operatorname{diag}(1,-1,-1,-1)$ is the metric tensor, and $u^{\mu}$ is the 4 -vector of fluid velocity. Noticing that

$$
u^{0}=\cosh y, \quad u^{1}=\sinh y,
$$

where $y$ is the ordinary rapidity of fluid, the $1+1$ expansion of liquid obeys equations

$$
\begin{gathered}
\frac{e^{2 y}-1}{2} \frac{\partial(\varepsilon+p)}{\partial z^{+}}+e^{2 y}(\varepsilon+p) \frac{\partial y}{\partial z^{+}}+\frac{1-e^{-2 y}}{2} \frac{\partial(\varepsilon+p)}{\partial z^{-}} \\
+e^{-2 y}(\varepsilon+p) \frac{\partial y}{\partial z^{-}}+\frac{\partial p}{\partial z^{+}}-\frac{\partial p}{\partial z^{-}}=0, \\
\frac{e^{2 y}+1}{2} \frac{\partial(\varepsilon+p)}{\partial z^{+}}+e^{2 y}(\varepsilon+p) \frac{\partial y}{\partial z^{+}}+\frac{1+e^{-2 y}}{2} \frac{\partial(\varepsilon+p)}{\partial z^{-}} \\
-e^{-2 y}(\varepsilon+p) \frac{\partial y}{\partial z^{-}}-\frac{\partial p}{\partial z^{+}}-\frac{\partial p}{\partial z^{-}}=0,
\end{gathered}
$$

where $z^{ \pm}=t \pm z=x^{0} \pm x^{1}=\tau e^{ \pm \eta}$ is the lightcone coordinates, $\tau=\sqrt{z^{+} z^{-}}$is the proper time, and $\eta=$ $1 / 2 \ln \left(z^{+} / z^{-}\right)$is the space-time rapidity of fluid.

In case of vanishing chemical potential,

$$
\varepsilon+p=T s, \quad \mathrm{~d} \varepsilon=T \mathrm{~d} s, \quad \mathrm{~d} p=s \mathrm{~d} T,
$$

where $T$ and $s$ are, respectively, the temperature and entropy density of fluid. From the above two equations, we can get relation

$$
\frac{\partial e^{-\theta+y}}{\partial z^{+}}=\frac{\partial e^{-\theta-y}}{\partial z^{-}}
$$

where

$$
\theta=\ln \left(\frac{T_{0}}{T}\right)
$$

where $T_{0}$ is an arbitrary initial temperature scale. Equation (6) means the existence of a potential $\phi\left(z^{+}, z^{-}\right)$satisfying

$$
\frac{\partial \phi\left(z^{+}, z^{-}\right)}{\partial z^{\mp}}=u^{ \pm} T=T_{0} e^{-\theta \pm y}
$$

where $u^{ \pm}=u^{0} \pm u^{1}=e^{ \pm y}$ are the light-cone variables of fluid velocity. In this way, (6) is automatically fulfilled.

Equation (4) is a complicated, nonlinear, and coupled one. In order to solve it, one introduces Khalatnikov potential

$$
\chi(\theta, y)=\phi\left(z^{+}, z^{-}\right)-z^{-} u^{+} T-z^{+} u^{-} T,
$$

where $z^{ \pm}$are the functions of $(\theta, y)$ implicitly defined by $\phi\left(z^{+}, z^{-}\right)$in (8) and can be expressed by Khalatnikov potential as

$$
z^{ \pm}(\theta, y)=\frac{1}{2 T_{0}} e^{\theta \pm y}\left(\frac{\partial \chi}{\partial \theta} \pm \frac{\partial \chi}{\partial y}\right)
$$

Following from this relation, one can get

$$
\begin{gathered}
\tau(\theta, y)=\frac{e^{\theta}}{2 T_{0}} \sqrt{\left(\frac{\partial \chi}{\partial \theta}\right)^{2}-\left(\frac{\partial \chi}{\partial y}\right)^{2}}, \\
\eta(\theta, y)=y+\frac{1}{2} \ln \left(\frac{\partial \chi / \partial \theta+\partial \chi / \partial y}{\partial \chi / \partial \theta-\partial \chi / \partial y}\right) .
\end{gathered}
$$

From (4), we can get the equation of $\chi$ as

$$
\frac{\partial^{2} \chi(\theta, y)}{\partial \theta^{2}}-[g(\theta)-1] \frac{\partial \chi(\theta, y)}{\partial \theta}-g(\theta) \frac{\partial^{2} \chi(\theta, y)}{\partial y^{2}}=0
$$

where

$$
g(\theta)=-\frac{1}{s} \frac{\mathrm{d} s}{\mathrm{~d} \theta}=\frac{1}{c_{s}^{2}(\theta)},
$$

where $c(\theta)$ is the speed of sound. Equation (12) is now a linear second-order partial differential equation, which works for any form of $g(\theta)$.

Experimental investigations have shown that the speed of sound is a constant of about $c_{s}=0.35$ or $g=8.16$, which is almost independent of interaction energy and system [2932]. In this case, we can take

$$
\chi(\theta, y)=e^{((g-1) / 2) \theta} Z(\theta, y) .
$$

Equation (12) becomes

$$
\frac{\partial^{2} Z(\theta, y)}{\partial \theta^{2}}-g \frac{\partial^{2} Z(\theta, y)}{\partial y^{2}}-\frac{(g-1)^{2}}{4} Z(\theta, y)=0 .
$$

The Green's function $G(\theta, y)$ of $Z(\theta, y)$ meets equation

$$
\frac{\partial^{2} G(\theta, y)}{\partial \theta^{2}}-g \frac{\partial^{2} G(\theta, y)}{\partial y^{2}}-\frac{(g-1)^{2}}{4} G(\theta, y)=\delta(\theta) \delta(y) \text {. }
$$

It has solution

$G(\theta, y)$

$$
=\frac{1}{4 \sqrt{g}} \Theta\left(\theta-\frac{y}{\sqrt{g}}\right) \Theta\left(\theta+\frac{y}{\sqrt{g}}\right) I_{0}\left(\frac{g-1}{2} \sqrt{\theta^{2}-\frac{y^{2}}{g}}\right),
$$

where $\Theta$ is the Heaviside step function and $I_{0}$ is the 0th order modified Bessel function of the first kind. Thus, we arrive at

$$
\begin{aligned}
\chi(\theta, y)= & e^{((g-1) / 2) \theta} \int \mathrm{d} y^{\prime} \int \mathrm{d} \theta^{\prime} G\left(\theta-\theta^{\prime}, y-y^{\prime}\right) F\left(\theta^{\prime}, y^{\prime}\right) \\
= & \frac{e^{((g-1) / 2) \theta}}{4 \sqrt{g}} \int \mathrm{d} y^{\prime} \int_{0}^{\theta-\left(y-y^{\prime}\right) / \sqrt{g}} \mathrm{~d} \theta^{\prime} F\left(\theta^{\prime}, y^{\prime}\right) \\
& \times I_{0}\left(\frac{g-1}{2} \sqrt{\left.\left(\theta-\theta^{\prime}\right)^{2}-\frac{\left(y-y^{\prime}\right)^{2}}{g}\right)},\right.
\end{aligned}
$$


where $F\left(\theta^{\prime}, y^{\prime}\right)$ stands for the distributions of sources of hydrodynamic flow.

In heavy ion collisions at high energy, owing to the violent compression of collision system along beam direction, the initial pressure gradient of created matter in this direction is very large. By contrast, the effect of initial flow of sources is negligible. The motion of liquid is mainly dominated by the following evolution. The typical example reflecting such fact is the Landau hydrodynamic model $[14,15]$, where the fluid is assumed initially at rest. In this evolution-dominated case, the source function may take the form as $[12,15,33]$

$$
F\left(\theta^{\prime}, y^{\prime}\right)=C^{\prime} e^{(-(g+1) / 2) \theta^{\prime}} \Theta\left(\theta^{\prime}\right) \delta\left(y^{\prime}\right),
$$

where $C^{\prime}$ is a constant. Inserting it into (18), we finally obtain the solution

$$
\chi(\theta, y)=C^{\prime} e^{-\theta} \int_{y / \sqrt{g}}^{\theta} \mathrm{d} \theta^{\prime} e^{((g+1) / 2) \theta^{\prime}} I_{0}\left(\frac{g-1}{2} \sqrt{\theta^{\prime 2}-\frac{y^{2}}{g}}\right) .
$$

\section{The Rapidity Distributions in High Energy Heavy Ion Collisions}

As an application of the Khalatnikov potential of (20), we will now derive the rapidity distribution of the charged particles produced in high energy heavy ion collisions. To this end, we first evaluate the entropy distribution at freeze-out temperature $T_{\mathrm{FO}}=T_{0} e^{-\theta_{\mathrm{FO}}}$ as a function of rapidity $y$.

The entropy distribution at freeze-out temperature is defined as the amount of entropy flowing through the hypersurface with a fixed temperature $T_{\mathrm{FO}}$ in a unit rapidity interval. It has the form as [12]

$$
\frac{\mathrm{d} S}{\mathrm{~d} y}=s_{\mathrm{FO}} \frac{u^{\mu} \mathrm{d} \lambda_{\mu}}{\mathrm{d} y}=s_{\mathrm{FO}} u^{\mu} n_{\mu} \frac{\mathrm{d} \lambda}{\mathrm{d} y}
$$

where $n^{\mu}$ is the 4-dimensional unit vector of the hypersurface

$$
n^{\mu} n_{\mu}=n^{+} n^{-}=1
$$

$\mathrm{d} \lambda$ is the space-like slab element along hypersurface with fixed temperature $T_{\mathrm{FO}}$, which is defined as $\mathrm{d} \lambda^{\mu}=\mathrm{d} \lambda n^{\mu}$ meeting

$$
(\mathrm{d} \lambda)^{2}=\mathrm{d} \lambda^{\mu} \mathrm{d} \lambda_{\mu}=-\mathrm{d} z_{\mathrm{FO}}^{+} \mathrm{d} z_{\mathrm{FO}}^{-}
$$

where the minus sign accounts for the space-like characteristic of $\mathrm{d} \lambda$.
In the $(\theta, y)$-base, the fixed-temperature hypersurface can be conveniently defined by

$$
\begin{aligned}
& \tau_{\mathrm{FO}}(y)=\tau\left(\theta_{\mathrm{FO}}, y\right), \\
& \eta_{\mathrm{FO}}(y)=\eta\left(\theta_{\mathrm{FO}}, y\right) .
\end{aligned}
$$

The tangential vector of the hypersurface is

$$
\begin{aligned}
& t^{+}(y) \equiv z_{\mathrm{FO}}^{+\prime}(y)=\left(\tau_{\mathrm{FO}}^{\prime}+\eta_{\mathrm{FO}}^{\prime} \tau_{\mathrm{FO}}\right) e^{\eta_{\mathrm{FO}}}, \\
& t^{-}(y) \equiv z_{\mathrm{FO}}^{-\prime}(y)=\left(\tau_{\mathrm{FO}}^{\prime}-\eta_{\mathrm{FO}}^{\prime} \tau_{\mathrm{FO}}\right) e^{-\eta_{\mathrm{FO}}},
\end{aligned}
$$

where the primes represent the derivatives with regard to $y$. According to definitions, we have

$$
n^{\mu}(y) t_{\mu}(y)=\frac{1}{2}\left[n^{+}(y) t^{-}(y)+n^{-}(y) t^{+}(y)\right]=0 .
$$

Owing to (25), the above equation turns into

$$
n^{+}(y)\left(\eta_{\mathrm{FO}}^{\prime} \tau_{\mathrm{FO}}-\tau_{\mathrm{FO}}^{\prime}\right) e^{-\eta_{\mathrm{FO}}}=n^{-}(y)\left(\eta_{\mathrm{FO}}^{\prime} \tau_{\mathrm{FO}}+\tau_{\mathrm{FO}}^{\prime}\right) e^{\eta_{\mathrm{FO}}} .
$$

This equation together with (22) gives

$$
\begin{aligned}
& n^{+}(y)=\sqrt{\frac{\eta_{\mathrm{FO}}^{\prime} \tau_{\mathrm{FO}}+\tau_{\mathrm{FO}}^{\prime}}{\eta_{\mathrm{FO}}^{\prime} \tau_{\mathrm{FO}}-\tau_{\mathrm{FO}}^{\prime}}} e^{\eta_{\mathrm{FO}}}, \\
& n^{-}(y)=\sqrt{\frac{\eta_{\mathrm{FO}}^{\prime} \tau_{\mathrm{FO}}-\tau_{\mathrm{FO}}^{\prime}}{\eta_{\mathrm{FO}}^{\prime} \tau_{\mathrm{FO}}+\tau_{\mathrm{FO}}^{\prime}}} e^{-\eta_{\mathrm{FO}}} .
\end{aligned}
$$

Equation (25) translates (23) into

$$
\mathrm{d} \lambda=\sqrt{\eta_{\mathrm{FO}}^{\prime 2} \tau_{\mathrm{FO}}^{2}-\tau_{\mathrm{FO}}^{\prime 2}} \mathrm{~d} y .
$$

Making use of (28), we obtain

$$
\begin{array}{r}
u^{\mu} n_{\mu}=\frac{1}{\sqrt{\eta_{\mathrm{FO}}^{\prime 2} \tau_{\mathrm{FO}}^{2}-\tau_{\mathrm{FO}}^{\prime 2}}}\left[\eta_{\mathrm{FO}}^{\prime} \tau_{\mathrm{FO}} \cosh \left(\eta_{\mathrm{FO}}-y\right)\right. \\
\left.+\tau_{\mathrm{FO}}^{\prime} \sinh \left(\eta_{\mathrm{FO}}-y\right)\right] .
\end{array}
$$

Using (29) and (30), (21) reads

$$
\frac{\mathrm{d} S}{\mathrm{~d} y}=s_{\mathrm{FO}}\left[\eta_{\mathrm{FO}}^{\prime} \tau_{\mathrm{FO}} \cosh \left(\eta_{\mathrm{FO}}-y\right)+\tau_{\mathrm{FO}}^{\prime} \sinh \left(\eta_{\mathrm{FO}}-y\right)\right] .
$$

Furthermore, known from (10),

$$
\begin{aligned}
& \cosh (\eta-y)=\frac{e^{\theta}}{2 \tau T_{0}} \frac{\partial \chi(\theta, y)}{\partial \theta} \\
& \sinh (\eta-y)=\frac{e^{\theta}}{2 \tau T_{0}} \frac{\partial \chi(\theta, y)}{\partial y}
\end{aligned}
$$

Deduced from (11), 


$$
\begin{gathered}
\tau_{\mathrm{FO}}^{\prime}=\left.\frac{e^{\theta}}{2 T_{0}} \frac{(\partial \chi / \partial \theta)\left(\partial^{2} \chi / \partial \theta \partial y\right)-(\partial \chi / \partial y)\left(\partial^{2} \chi / \partial y^{2}\right)}{\sqrt{(\partial \chi / \partial \theta)^{2}-(\partial \chi / \partial y)^{2}}}\right|_{\theta=\theta_{\mathrm{FO}}}, \\
\eta_{\mathrm{FO}}^{\prime}=\left.\frac{(\partial \chi / \partial \theta)^{2}-(\partial \chi / \partial y)^{2}-(\partial \chi / \partial y)\left(\partial^{2} \chi / \partial \theta \partial y\right)+(\partial \chi / \partial \theta)\left(\partial^{2} \chi / \partial y^{2}\right)}{(\partial \chi / \partial \theta)^{2}-(\partial \chi / \partial y)^{2}}\right|_{\theta=\theta_{\mathrm{FO}}} .
\end{gathered}
$$

These two equations make (31) become

$$
\frac{\mathrm{d} S}{\mathrm{~d} y}=\left.\frac{s_{\mathrm{FO}}}{2 T_{\mathrm{FO}} g}\left[\frac{\partial^{2} \chi(\theta, y)}{\partial \theta^{2}}+\frac{\partial \chi(\theta, y)}{\partial \theta}\right]\right|_{\theta=\theta_{\mathrm{FO}}}
$$

For evolution-dominated hydrodynamics, substituting (20) into the above equation, we acquire

$$
\begin{aligned}
\frac{\mathrm{d} S}{\mathrm{~d} y}=\frac{s_{\mathrm{FO}}(g-1) C^{\prime}}{4 g T_{\mathrm{FO}}} e^{((g-1) / 2) \theta_{\mathrm{FO}}} \\
\times\left[I_{0}\left(\frac{g-1}{2} \sqrt{\theta_{\mathrm{FO}}^{2}-\frac{y^{2}}{g}}\right)\right. \\
\left.+\frac{\theta_{\mathrm{FO}}}{\sqrt{\theta_{\mathrm{FO}}^{2}-y^{2} / g}} I_{1}\left(\frac{g-1}{2} \sqrt{\theta_{\mathrm{FO}}^{2}-\frac{y^{2}}{g}}\right)\right],
\end{aligned}
$$

where $I_{1}$ is the 1st order modified Bessel function of the first kind. $\theta_{\mathrm{FO}}=\ln \left(T_{0} / T_{\mathrm{FO}}\right)$ is related to the initial temperature of fluid and is therefore dependent on the incident energy and collision centrality. Since $T_{0}$ should not vary very much with centrality cuts, we can expect that $\theta_{\mathrm{FO}}$ would approximately maintain a constant or at least depend weakly on collision centrality for a given incident energy.

As the entropy is proportional to the number of produced charged particles, we obtain the rapidity distribution

$$
\begin{aligned}
& \frac{\mathrm{d} N_{\text {Fluid }}\left(b, \sqrt{s_{\mathrm{NN}}}, y\right)}{\mathrm{d} y} \\
&=C\left(b, \sqrt{s_{\mathrm{NN}}}\right)\left[I_{0}\left(\frac{g-1}{2} \sqrt{\theta_{\mathrm{FO}}^{2}-\frac{y^{2}}{g}}\right)\right. \\
&\left.+\frac{\theta_{\mathrm{FO}}}{\sqrt{\theta_{\mathrm{FO}}^{2}-y^{2} / g}} I_{1}\left(\frac{g-1}{2} \sqrt{\theta_{\mathrm{FO}}^{2}-\frac{y^{2}}{g}}\right)\right],
\end{aligned}
$$

where $C\left(b, \sqrt{s_{\mathrm{NN}}}\right)$, independent of rapidity $y$, is an overall normalization constant. $b$ is the impact parameter, and $\sqrt{s_{\mathrm{NN}}}$ is the center-of-mass energy per pair of nucleons.

\section{Comparison with Experimental Measurements and the Rapidity Distributions of Leading Particles}

Figure 1 shows the rapidity distributions for $\pi^{+}, \pi^{-}, K^{+}, K^{-}$, $p$, and $\bar{p}$ produced in central Au-Au collisions at $\sqrt{s_{\mathrm{NN}}}=$ $200 \mathrm{GeV}$. The scattered symbols are the experimental data [24-26]. The solid curves are the theoretical results from (36). In calculations, the parameter $\theta_{\mathrm{FO}}$ takes the value of $\theta_{\mathrm{FO}}=2.23$. We can see from this figure that, except for proton $p$, (36) fits well with experimental measurements. For proton $p$, experimental data show an evident uplift in the rapidity interval between $y=2.0$ and 3.0. This may result from parts of leading particles, which are free from the description of hydrodynamics. Hence, in order to match up with experimental data, we should take these leading particles into account separately.

Considering that, for a given incident energy, the leading particles in each time of nucleus-nucleus collisions have approximately the same amount of energy, then, according to the central limit theorem $[34,35]$, the leading particles should follow the Gaussian rapidity distribution. That is,

$$
\begin{aligned}
& \frac{\mathrm{d} N_{\text {Lead }}\left(b, \sqrt{s_{\mathrm{NN}}}, y\right)}{\mathrm{d} y} \\
& =\frac{N_{\text {Lead }}\left(b, \sqrt{s_{\mathrm{NN}}}\right)}{\sqrt{2 \pi} \sigma} \exp \left\{-\frac{\left[|y|-y_{0}\left(b, \sqrt{s_{\mathrm{NN}}}\right)\right]^{2}}{2 \sigma^{2}}\right\},
\end{aligned}
$$

where $y_{0}\left(b, \sqrt{s_{\mathrm{NN}}}\right)$ and $\sigma$ are, respectively, the central position and width of distribution. In fact, as is known to all, the rapidity distribution of any charged particles produced in heavy ion collisions can be well represented by Gaussian form ([24-26]; also confer the shapes of the curves in Figure 1). It is obvious that $y_{0}\left(b, \sqrt{s_{\mathrm{NN}}}\right)$ should increase with incident energy and centrality cut. However, $\sigma$ should not apparently depend on them. This is due to the fact that the relative energy differences among leading particles should not be too much for different incident energies and centrality cuts. $N_{\text {Lead }}\left(b, \sqrt{s_{\mathrm{NN}}}\right)$ in (37) is the number of leading particles. It is a function of energy and centrality.

It is well known that, in nucleon-nucleon, such as $p$ $p$, collisions, there are two leading particles. One is in projectile fragmentation region, and the other is in target fragmentation region. Then, in nucleus-nucleus collisions, the leading particles should be those nucleons which participate in collisions, the so-called participants, which locate 
TABLE 1: The mean numbers of total participants $\bar{N}_{\text {Part }}$ and the central positions $y_{0}$ of Gaussian rapidity distribution in different centrality $\mathrm{Au}-\mathrm{Au}$ and $\mathrm{Cu}-\mathrm{Cu}$ collisions at $\sqrt{s_{\mathrm{NN}}}=200 \mathrm{GeV}$. The numbers with and without errors are, respectively, the results given by PHOBOS Collaboration at BNL-RHIC [27] and (39).

\begin{tabular}{lccccccccc}
\hline Centrality cut (\%) & $0-3$ & $3-6$ & $6-10$ & $10-15$ & $15-20$ & $20-25$ & $25-30$ & $30-35$ & $35-40$ \\
\hline $\bar{N}_{\text {Part }}(\mathrm{Au}-\mathrm{Au})$ & 359.44 & 324.50 & 288.74 & 248.96 & 210.98 & 178.24 & 149.78 & 124.92 & 103.22 \\
& $361 \pm 11$ & $331 \pm 10$ & $297 \pm 9$ & $255 \pm 8$ & $215 \pm 7$ & $180 \pm 7$ & $150 \pm 6$ & $124 \pm 6$ & $101 \pm 6$ \\
\hline $\bar{N}_{\text {Part }}(\mathrm{Cu}-\mathrm{Cu})$ & 109.92 & 99.76 & 89.00 & 76.70 & 64.74 & 54.40 & 45.40 & 37.62 & 30.88 \\
& $108 \pm 4$ & $101 \pm 3$ & $91 \pm 3$ & $79 \pm 3$ & $67 \pm 3$ & $57 \pm 3$ & $48 \pm 3$ & $40 \pm 3$ & $33 \pm 3$ \\
\hline$y_{0}(\mathrm{Au}-\mathrm{Au})$ & 2.63 & 2.67 & 2.70 & 2.72 & 2.78 & 2.81 & 2.96 & 2.97 & 3.05 \\
\hline$y_{0}(\mathrm{Cu}-\mathrm{Cu})$ & 2.75 & 2.78 & 2.80 & 2.93 & 2.94 & 2.95 & 2.96 & 2.97 & 3.05 \\
\hline
\end{tabular}

TABLE 2: The mean numbers of total participants $\bar{N}_{\text {Part }}$ and the central positions $y_{0}$ of Gaussian rapidity distribution in different centrality $\mathrm{Pb}-\mathrm{Pb}$ collisions at $\sqrt{s_{\mathrm{NN}}}=2.76 \mathrm{TeV}$. The numbers with and without errors are, respectively, the results given by ALICE Collaboration at CERN-LHC [28] and (39).

\begin{tabular}{lcccc}
\hline Centrality cut $(\%)$ & $0-5$ & $5-10$ & $10-20$ & $20-30$ \\
\hline $\bar{N}_{\text {Part }}(\mathrm{Pb}-\mathrm{Pb})$ & 381.56 & 327.70 & 261.90 & 189.78 \\
& $383 \pm 3$ & $330 \pm 5$ & $261 \pm 4$ & $186 \pm 4$ \\
\hline$y_{0}(\mathrm{~Pb}-\mathrm{Pb})$ & 3.38 & 3.41 & 3.44 & 3.48 \\
\hline
\end{tabular}

separately at projectile and target fragmentation regions. For collisions between two identical nuclei, each nucleus should have about the same number of participants. Hence, the number of leading particles appearing in projectile or target fragmentation region should be

$$
N_{\text {Lead }}\left(b, \sqrt{s_{\mathrm{NN}}}\right)=\frac{N_{\text {Part }}\left(b, \sqrt{s_{\mathrm{NN}}}\right)}{2},
$$

where $N_{\text {Part }}\left(b, \sqrt{s_{\mathrm{NN}}}\right)$ is the number of total participants in two nuclei, which can be evaluated by formula $[36,37]$

$$
N_{\text {Part }}\left(b, \sqrt{s_{\mathrm{NN}}}\right)=\int n_{\text {Part }}\left(b, \sqrt{s_{\mathrm{NN}}}, s\right) \mathrm{d}^{2} s,
$$

where $s$ is the coordinates in the overlap region measured from the center of one nucleus. The integrand in above equation:

$$
\begin{aligned}
n_{\text {Part }} & \left(b, \sqrt{s_{\mathrm{NN}}}, s\right) \\
= & T_{A}(s)\left\{1-\exp \left[-\sigma_{\mathrm{NN}}^{\mathrm{in}}\left(\sqrt{s_{\mathrm{NN}}}\right) T_{B}(s-b)\right]\right\} \\
& +T_{B}(s-b)\left\{1-\exp \left[-\sigma_{\mathrm{NN}}^{\mathrm{in}}\left(\sqrt{s_{\mathrm{NN}}}\right) T_{A}(s)\right]\right\},
\end{aligned}
$$

where $\sigma_{\mathrm{NN}}^{\text {in }}\left(\sqrt{s_{\mathrm{NN}}}\right)$ is the inelastic nucleon-nucleon crosssection. It increases slowly with energy. For example, for $\sqrt{s_{\mathrm{NN}}}=200 \mathrm{GeV}, \sigma_{\mathrm{NN}}^{\text {in }}=42 \mathrm{mb}$ [38], and, for $\sqrt{s_{\mathrm{NN}}}=$ $2.76 \mathrm{TeV}, \sigma_{\mathrm{NN}}^{\mathrm{in}}=64 \pm 5 \mathrm{mb}$ [39]. The subscripts $A$ and $B$ in the above equation denote the projectile and target nucleus, respectively. $T(s)$ is the thickness function defined as

$$
T(s)=\int \rho(s, z) \mathrm{d} z,
$$

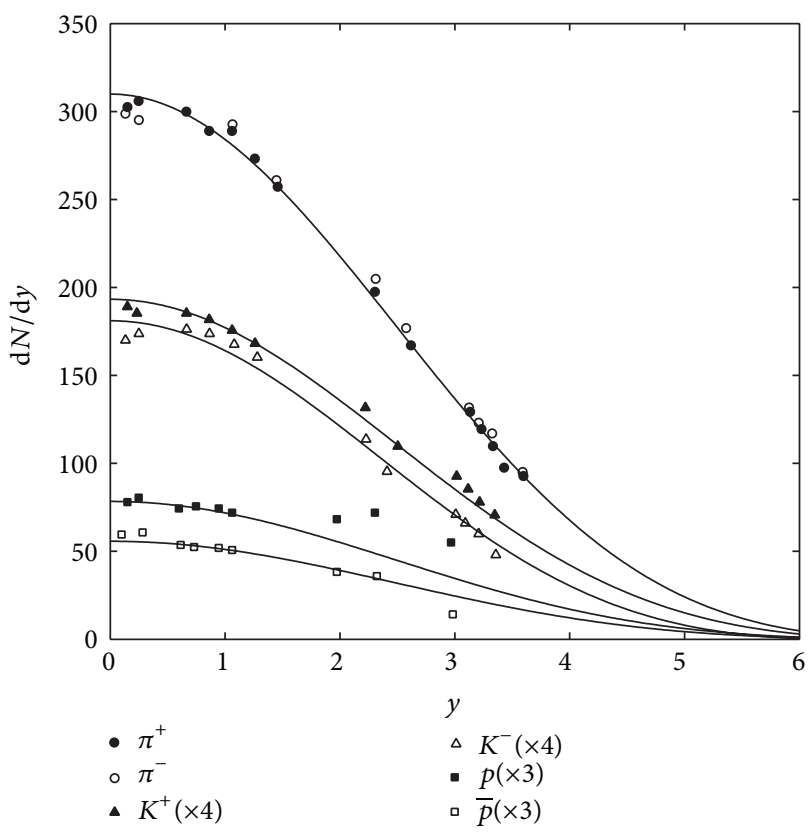

FIgURE 1: The rapidity distributions of specified charged particles in central Au-Au collisions at $\sqrt{s_{\mathrm{NN}}}=200 \mathrm{GeV}$. The scattered symbols are the experimental measurements [24-26]. The solid curves are the results from the evolution-dominated hydrodynamics of (36).

where

$$
\rho(r)=\frac{\rho_{0}}{1+\exp \left[\left(r-r_{0}\right) / a\right]}
$$

is the Woods-Saxon distribution of nuclear density. $a$ and $r_{0}$ are, respectively, the skin depth and radius of nucleus. In this paper, they take the values of $a=0.54 \mathrm{fm}$ and $r_{0}=$ $1.12 A^{1 / 3}-0.86 A^{-1 / 3} \mathrm{fm}[36]$, where $A$ is the mass number of nucleus.

Tables 1 and 2 show the mean numbers of total participants in different centrality $\mathrm{Au}-\mathrm{Au}$ and $\mathrm{Cu}-\mathrm{Cu}$ collisions at $\sqrt{s_{\mathrm{NN}}}=200 \mathrm{GeV}$ and $\mathrm{Pb}-\mathrm{Pb}$ collisions at $\sqrt{s_{\mathrm{NN}}}=2.76 \mathrm{TeV}$. The numbers with and without errors are those given by experiments [27, 28] and (39), respectively. Due to space constraints, Table 1 only shows the numbers in the first nine centrality cuts. It can be seen that both sets of numbers coincide well. 

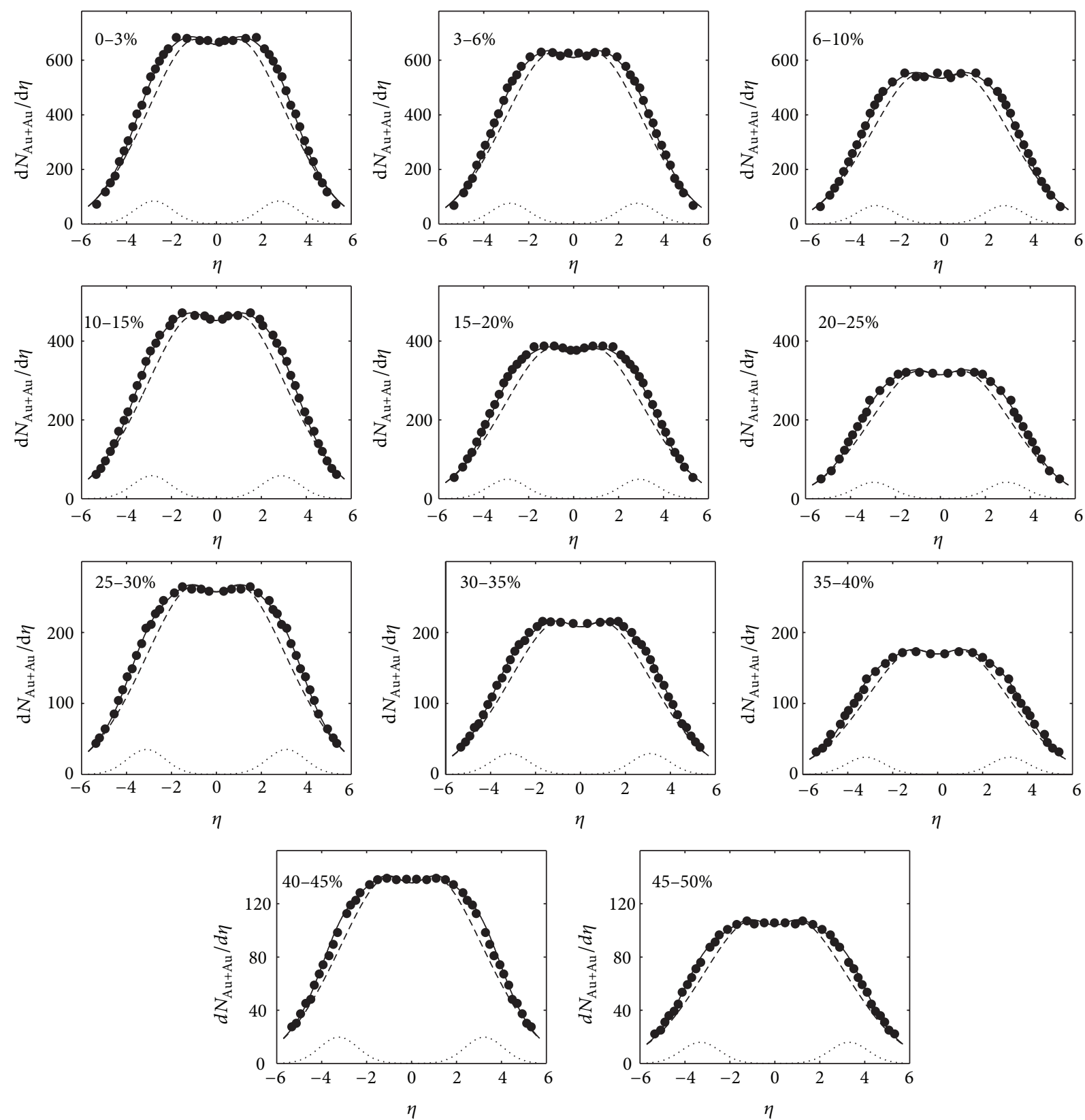

FIGURE 2: The pseudorapidity distributions of the charged particles produced in different centrality Au-Au collisions at $\sqrt{s_{\mathrm{NN}}}=200 \mathrm{GeV}$. The solid dots are the experimental measurements [27]. The dashed curves are the results from evolution-dominated hydrodynamics of (36). The dotted curves are the results from leading particles of (37). The solid curves are the sums of dashed and dotted ones.

Having the rapidity distributions of (36) and (37), the pseudorapidity distribution measured in experiments can be expressed as [40]

$$
\begin{gathered}
\frac{\mathrm{d} N\left(b, \sqrt{s_{\mathrm{NN}}}, \eta\right)}{\mathrm{d} \eta}=\sqrt{1-\frac{m^{2}}{m_{T}^{2} \cosh ^{2} y}} \frac{\mathrm{d} N\left(b, \sqrt{s_{\mathrm{NN}}}, y\right)}{\mathrm{d} y}, \\
y=\frac{1}{2} \ln \left[\frac{\sqrt{p_{T}^{2} \cosh ^{2} \eta+m^{2}}+p_{T} \sinh \eta}{\sqrt{p_{T}^{2} \cosh ^{2} \eta+m^{2}}-p_{T} \sinh \eta}\right],
\end{gathered}
$$

where $p_{T}$ is the transverse momentum, $m_{T}=\sqrt{m^{2}+p_{T}^{2}}$ is the transverse mass, and

$$
\begin{aligned}
& \frac{\mathrm{d} N\left(b, \sqrt{s_{\mathrm{NN}}}, y\right)}{\mathrm{d} y} \\
& =\frac{\mathrm{d} N_{\text {Fluid }}\left(b, \sqrt{s_{\mathrm{NN}}}, y\right)}{\mathrm{d} y}+\frac{\mathrm{d} N_{\text {Lead }}\left(b, \sqrt{s_{\mathrm{NN}}}, y\right)}{\mathrm{d} y}
\end{aligned}
$$

is the total rapidity distribution from both fluid evolution and leading particles.

Substituting (45) into (43), we can get the pseudorapidity distributions of charged particles. Figures 2, 3, and 4 show 

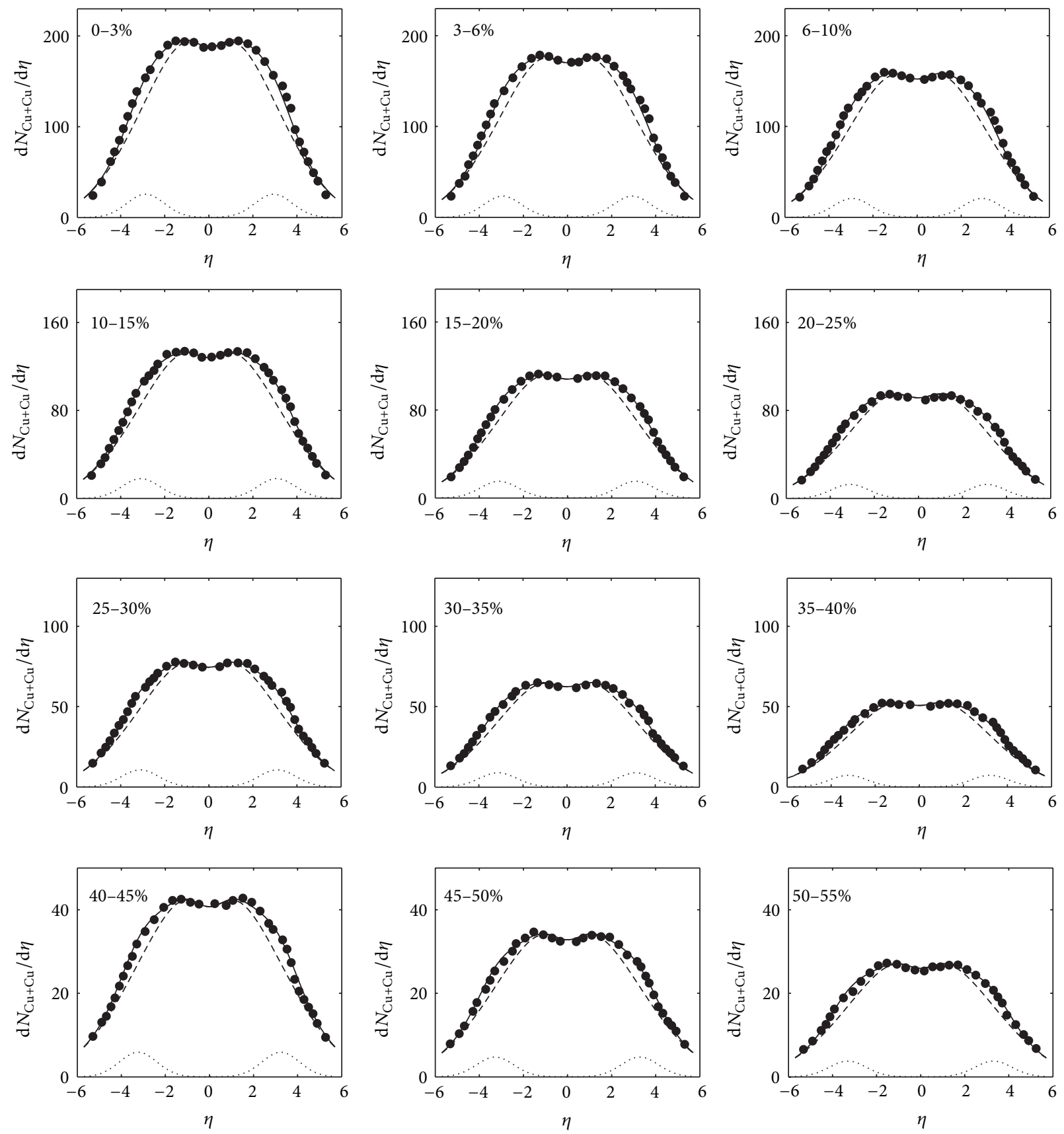

Figure 3: The pseudorapidity distributions of the charged particles produced in different centrality Cu-Cu collisions at $\sqrt{s_{\mathrm{NN}}}=200 \mathrm{GeV}$. The solid dots are the experimental measurements [27]. The dashed curves are the results from evolution-dominated hydrodynamics of (36). The dotted curves are the results from leading particles of (37). The solid curves are the sums of dashed and dotted ones.

such distributions in different centrality $\mathrm{Au}-\mathrm{Au}$ and $\mathrm{Cu}-$ $\mathrm{Cu}$ collisions at $\sqrt{s_{\mathrm{NN}}}=200 \mathrm{GeV}$ and $\mathrm{Pb}-\mathrm{Pb}$ collisions at $2.76 \mathrm{TeV}$, respectively. The solid dots in figures are the experimental measurements $[27,28]$. The dashed curves are the results got from evolution-dominated hydrodynamics of (36). The dotted curves are the results obtained from leading particles of (37). The solid curves are the results achieved from (45), that is, the sums of dashed and dotted curves. It can be seen that the theoretical results are well consistent with experimental measurements.
In calculations, the parameter $\theta_{\mathrm{FO}}$ in (36) takes the values of 2.80 in the first three centrality cuts, 2.98 in the following six ones, and 3.17 in the last two ones in Au-Au collisions. In $\mathrm{Cu}-\mathrm{Cu}$ collisions, $\theta_{\mathrm{FO}}$ takes the value of 2.95 in the first three centrality cuts, 3.15 in the following six ones, and 3.53 in the last three ones. In $\mathrm{Pb}-\mathrm{Pb}$ collisions, $\theta_{\mathrm{FO}}$ takes the value of 5.85 for the first two centrality cuts and 6.04 for the second two ones. It can be seen that $\theta_{\mathrm{FO}}$ increases with incident energy and centrality cut. The width parameter $\sigma$ in (37) takes a constant of 0.85 for all three kinds of collision systems in 

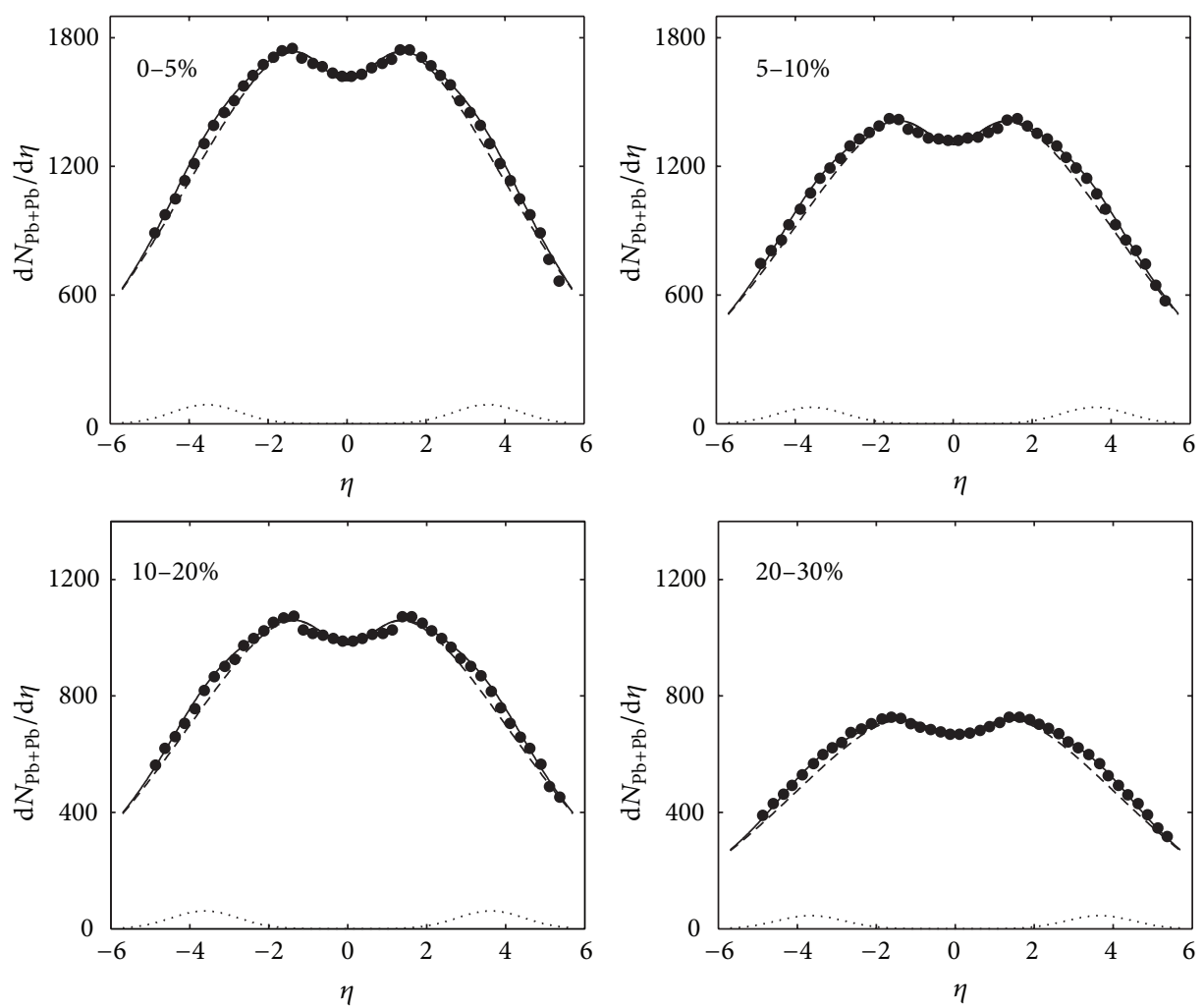

FIGURE 4: The pseudorapidity distributions of the charged particles produced in different centrality $\mathrm{Pb}-\mathrm{Pb}$ collisions at $\sqrt{\mathcal{S}_{\mathrm{NN}}}=2.76 \mathrm{TeV}$. The solid dots are the experimental measurements [28]. The dashed curves are the results from evolution-dominated hydrodynamics of (36). The dotted curves are the results from leading particles of (37). The solid curves are the sums of dashed and dotted ones.

different centrality cuts. As the analyses given above, $\sigma$ is independent of incident energy and centrality cut. What is more, it is also independent of collision system. The center parameter $y_{0}$ in (37) takes the values as listed in Tables 1 and 2. As stated early, $y_{0}$ increases with energy and centrality cut. From Table 1, we can see that, for a given incident energy and centrality cut, $y_{0}$ decreases with increasing nucleus size. This can be understood if we notice the fact that the larger the nucleus size, the more the collisions that the participants will undergo. Hence, the final leading particles will lose more energy or have smaller $y_{0}$. The fitting value of $y_{0}=2.63$ in the top 3\% most central Au-Au collisions is in accordance with the experimental observation shown in Figure 1, which indicates that the leading particles are mainly in the range between $y=2$ and 3. Experimental investigations also have shown that [41], in the top 5\% most central Au-Au collisions at $\sqrt{s_{\mathrm{NN}}}=200 \mathrm{GeV}$, the rapidity loss of participants is up to $\langle\delta y\rangle \approx 2.45$; then the leading particles should locate at

$$
y_{0}=y_{\text {beam }}-\langle\delta y\rangle=5.36-2.45=2.91 \text {. }
$$

Seeing the smaller centrality cut considered in our analysis, our above fitting result is also consistent with this measurement.

\section{Conclusions}

The charged particles produced in heavy ion collisions are divided into two parts. One is from the hot and dense matter created in collisions. The other is from leading particles.

Compared with the effect of pressure gradient, the effect of initial flow of the hot and dense matter is negligible. The motion of this matter is mainly governed by the evolution of fluid. This thus guarantees the rationality of evolutiondominated hydrodynamics. With the scheme of Khalatnikov potential, this theoretical model can be solved exactly, and the rapidity distribution of charged particles can be expressed in a simple analytical form in terms of 0th and 1st order modified Bessel function of the first kind with only two parameters $g=1 / c_{s}^{2}$ and $\theta_{\mathrm{FO}}=\ln \left(T_{0} / T_{\mathrm{FO}}\right) \cdot g$ takes the value from experiments. $\theta_{\mathrm{FO}}$ is fixed by fitting with experimental data.

For leading particles, we assume that the rapidity distribution of them possesses the Gaussian form with the normalization constant being equal to the number of participants, which can be figured out in theory. This assumption is based on the consideration that, for a given incident energy, the leading particles have about the same energy and coincides with the fact that any kind of charged particles takes on well the Gaussian form of rapidity distribution. It is interesting to notice that the width of Gaussian rapidity distribution $\sigma$ is irrelevant to the incident energy, centrality cut, and collision system. The fitting values of $y_{0}$, the central positions 
of Gaussian rapidity distribution, are in good accordance with experimental data.

Comparing with the experimental measurements made by BRAHMS and PHOBOS Collaboration at BNL-RHIC in $\mathrm{Au}-\mathrm{Au}$ and $\mathrm{Cu}-\mathrm{Cu}$ collisions at $\sqrt{s_{\mathrm{NN}}}=200 \mathrm{GeV}$ and by ALICE Collaboration at CERN-LHC in Pb-Pb collisions at $\sqrt{s_{\mathrm{NN}}}=2.76 \mathrm{TeV}$, we can see that the total contributions from both evolution-dominated hydrodynamics and leading particles are well consistent with experimental data.

\section{Conflict of Interests}

The authors declare that there is no conflict of interests regarding the publication of this paper.

\section{Acknowledgments}

This work is partly supported by the Transformation Project of Science and Technology of Shanghai Baoshan District with Grant no. CXY-2012-25 and by the Shanghai Leading Academic Discipline Project with Grant no. XTKX 2012.

\section{References}

[1] J.-Y. Ollitrault, "Anisotropy as a signature of transverse collective flow," Physical Review D, vol. 46, no. 1, pp. 229-245, 1992.

[2] S. S. Adler, S. Afanasiev, C. Aidala et al., "Elliptic flow of identified hadrons in $\mathrm{Au}+\mathrm{Au}$ collisions at $\sqrt{s_{\mathrm{NN}}}=200 \mathrm{GeV}$," Physical Review Letters, vol. 91, Article ID 182301, 2003.

[3] K. Aamodt, B. I. Abelev, M. Reicher et al., "Higher harmonic anisotropic flow measurements of charged particles in $\mathrm{Pb}-\mathrm{Pb}$ collisions at $\sqrt{s_{\mathrm{NN}}}=2.76 \mathrm{TeV}$," Physical Review Letters, vol. 107, Article ID 032301, 2011.

[4] P. A. Steinberg, "Bulk dynamics in heavy ion collisions," Nuclear Physics A, vol. 752, pp. 423-432, 2005.

[5] C. Y. Wong, "Landau hydrodynamics reexamined," Physical Review C, vol. 78, Article ID 054902, 2008.

[6] Z. J. Jiang, Q. G. Li, and H. L. Zhang, “The revised Landau hydrodynamic model and the pseudorapidity distributions of produced charged particles in high energy heavy ion collisions," Journal of Physics G: Nuclear and Particle Physics, vol. 40, Article ID 025101, 2013.

[7] A. Bialas and R. Peschanski, "Asymmetric $(1+1)$-dimensional hydrodynamics in high-energy collisions," Physical Review C: Nuclear Physics, vol. 83, no. 5, Article ID 054905, 2011.

[8] C. Gale, S. Jeon, and B. Schenke, "Hydrodynamic modeling of heavy-ion collisions," International Journal of Modern Physics A, vol. 28, no. 11, Article ID 1340011, 2013.

[9] E. K. G. Sarkisyan and A. S. Sakharov, "Relating multihadron production in hadronic and nuclear collisions," European Physical Journal C, vol. 70, no. 3, pp. 533-541, 2010.

[10] H. Song, S. A. Bass, U. Heinz, T. Hirano, and C. Shen, “200 A $\mathrm{GeV} \mathrm{Au}+\mathrm{Au}$ collisions serve a nearly perfect quark-gluon liquid," Physical Review Letters, vol. 106, no. 19, Article ID 192301, 2011.

[11] R. Rvblewski and W. Florkowski, "Highly anisotropic hydrodynamics in $3+1$ space-time dimensions," Physical Review C, vol. 85, Article ID 064901, 2012.
[12] G. Beuf, R. Peschanski, and E. N. Saridakis, "Entropy flow of a perfect fluid in $(1+1)$ hydrodynamics," Physical Review C: Nuclear Physics, vol. 78, no. 6, Article ID 064909, 2008.

[13] I. M. Khalatnikov, "Some questions of the relativistic hydrodynamics," Journal of Experimental and Theoretical Physics, vol. 27, pp. 529-541, 1954 (Russian).

[14] L. D. Landau, "On the multiple production of particles in fast particle collisions," Izvestiya Akademii Nauk SSSR, vol. 17, pp. 51-64, 1953 (Russian).

[15] S. Z. Belen'kji and L. D. Landau, "Hydrodynamic theory of multiple production of particles," Il Nuovo Cimento Series 10, vol. 3, no. 1, pp. 15-31, 1956.

[16] R. C. Hwa, "Statistical description of hadron constituents as a basis for the fluid model of high-energy collisions," Physical Review D, vol. 10, no. 7, pp. 2260-2268, 1974.

[17] J. D. Bjorken, "Highly relativistic nucleus-nucleus collisions: the central rapidity region," Physical Review D, vol. 27, no. 1, pp. 140151, 1983.

[18] T. Csörgö, N. I. Nagy, and M. Csanád, "New family of simple solutions of relativistic perfect fluid hydrodynamics," Physics Letters B, vol. 663, pp. 306-311, 2008.

[19] M. I. Nagy, T. Csörgö, and M. Csanád, "Detailed description of accelerating, simple solutions of relativistic perfect fluid hydrodynamics," Physical Review C, vol. 77, Article ID 024908, 2008.

[20] M. Csanád, M. I. Nagy, and T. Csörgö, "Similar final states from different initial states using new exact solutions of relativistic hydrodynamics," European Physical Journal ST, vol. 155, pp. 1926, 2008.

[21] A. Bialas, R. A. Janik, and R. Peschanski, "Unified description of Bjorken and Landau $1+1$ hydrodynamics," Physical Review C: Nuclear Physics, vol. 76, no. 5, Article ID 054901, 2007.

[22] M. S. Borshch and V. I. Zhdanov, "Exact solutions of the equations of relativistic hydrodynamics representing potential flows," Symmetry, Integrability and Geometry: Methods and Applications, vol. 3, article 116, 11 pages, 2007.

[23] M. Csanád, M. I. Nagy, and S. Lökös, "Exact solutions of relativistic perfect fluid hydrodynamics for a QCD Equation of State," European Physical Journal A, vol. 48, p. 173, 2012.

[24] M. Murray, "Scanning the phases of QCD with BRAHMS," Journal of Physics G: Nuclear and Particle Physics, vol. 30, pp. S667-S674, 2004.

[25] M. Murray, "Flavor dynamics," Journal of Physics G: Nuclear and Particle Physics, vol. 35, Article ID 044015, 2008.

[26] I. G. Bearden, D. Beavis, C. Besliu et al., "Charged meson rapidity distributions in central $\mathrm{Au}+\mathrm{Au}$ collisions at $\sqrt{s_{\mathrm{NN}}}=$ 200 GeV," Physical Review Letters, vol. 94, Article ID 162301, 2005.

[27] B. Alver, B. Back, M. Baker et al., "Charged-particle multiplicity and pseudorapidity distributions measured with the PHOBOS detector in $\mathrm{Au}+\mathrm{Au}, \mathrm{Cu}+\mathrm{Cu}, \mathrm{d}+\mathrm{Au}$, and $\mathrm{p}+\mathrm{p}$ collisions at ultrarelativistic energies," Physical Review C, vol. 83, Article ID 024913, 2011.

[28] E. Abbas, B. Abelev, J. Adam et al., "Centrality dependence of the pseudorapidity density distribution for charged particles in $\mathrm{Pb}-\mathrm{Pb}$ collisions at $\sqrt{s_{\mathrm{NN}}}=2.76 \mathrm{TeV}$," Physics Letters $B$, vol. 726, p. $610,2013$.

[29] A. Adare, S. Afanasiev, C. Aidala et al., "Scaling properties of azimuthal anisotropy in $\mathrm{Au}+\mathrm{Au}$ and $\mathrm{Cu}+\mathrm{Cu}$ collisions at $\sqrt{s_{\mathrm{NN}}}=200 \mathrm{GeV}$,' Physical Review Letters, vol. 98, p. 162301, 2007. 
[30] N. Armesto, N. Borghini, S. Jeon et al., "Heavy-ion collisions at the LHC-Last call for predictions," Journal of Physics G: Nuclear and Particle Physics, vol. 35, Article ID 054001, 2008.

[31] T. Mizoguchi, H. Miyazawa, and M. Biyajima, "A potential including the Heaviside function in the $1+1$ dimensional hydrodynamics by Landau: IIts basic properties and application to data at RHIC energies," European Physical Journal A, vol. 40, no. 1, pp. 99-108, 2009.

[32] S. Borsányi, G. Endrődi, Z. Fodor et al., "The QCD equation of state with dynamical quarks," Journal of High Energy Physics, vol. 77, p. 1, 2010.

[33] S. Amai, H. Fukuda, C. Iso, and M. Sato, "Hydrodynamical treatment of multiple meson production in high energy nucleon-nucleus collisions," Progress of Theoretical Physics, vol. 17, pp. 241-287, 1957.

[34] T. B. Li, The Mathematical Processing of Experiments, Science Press, Beijing, China, 1980, (Chinese).

[35] J. Voit, The Statistical Mechanics of Financial Markets, Springer, Berlin, Germany, 2005.

[36] Z.-J. Jiang, "The numbers of participants and nucleon-nucleon collisions in high-energy heavy-ion collisions," Acta Physica Sinica, vol. 56, no. 9, pp. 5191-5195, 2007 (Chinese).

[37] Z. J. Jiang, Y. F. Sun, and Q. G. Li, “The energy and centrality dependences of the pseudorapidity distributions of the charged particles in Au+Au collisions," International Journal of Modern Physics E, vol. 21, Article ID 1250002, 2012.

[38] B. B. Back, M. D. Baker, M. Ballintijn et al., "The PHOBOS perspective on discoveries at RHIC," Nuclear Physics A, vol. 757, pp. 28-101, 2005.

[39] K. Aamodt, A. Abrahantes Quintana, D. Adamová et al., "Centrality dependence of the charged-particle multiplicity density at midrapidity in $\mathrm{Pb}-\mathrm{Pb}$ collisions at $\sqrt{s_{\mathrm{NN}}}=2.76 \mathrm{TeV}$," Physical Review Letters, vol. 106, Article ID 032301, 2011.

[40] C. Y. Wong, Introduction To High Energy Heavy Ion Collisions, Press of Harbin Technology University, Harbin, China, 2002, (Chinese), English edition: World Scientific, Singapore, 1994.

[41] I. G. Bearden, D. Beavis, C. Besliu et al., "Nuclear stopping in $\mathrm{Au}+\mathrm{Au}$ collisions at $\sqrt{s_{\mathrm{NN}}}=200 \mathrm{GeV}$," Physical Review Letters, vol. 93, Article ID 102301, 2004. 

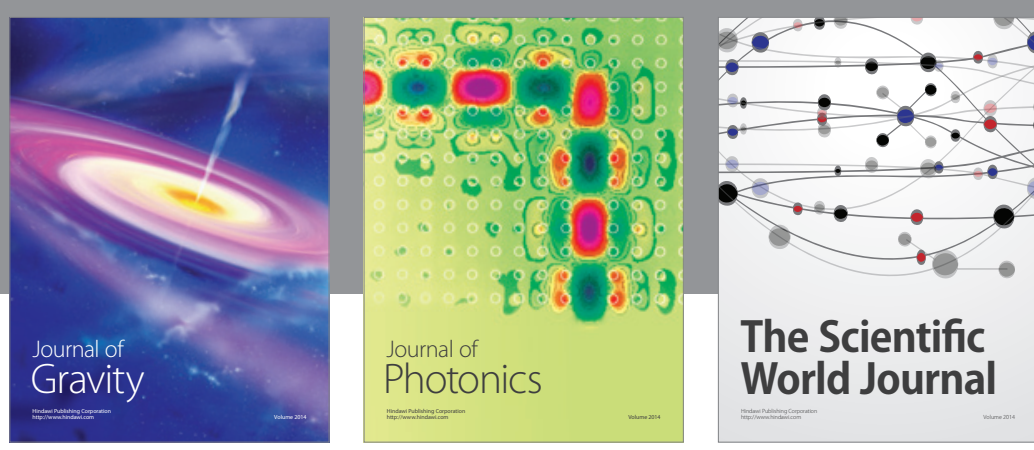

The Scientific World Journal
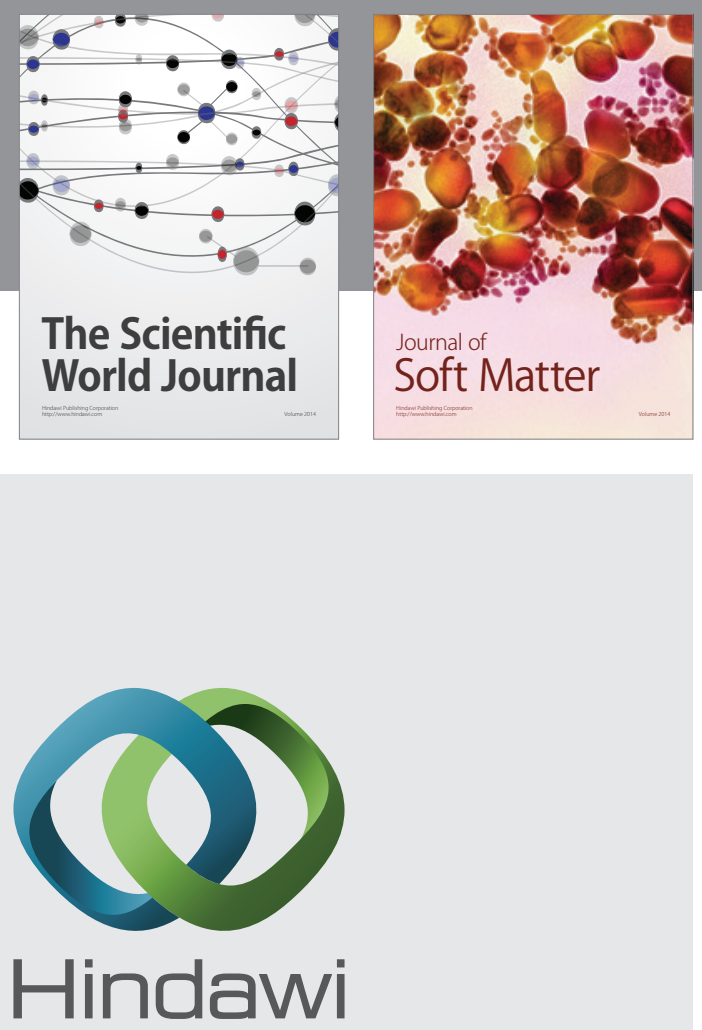

Submit your manuscripts at

http://www.hindawi.com

nternational Journal of

Statistical Mechanics
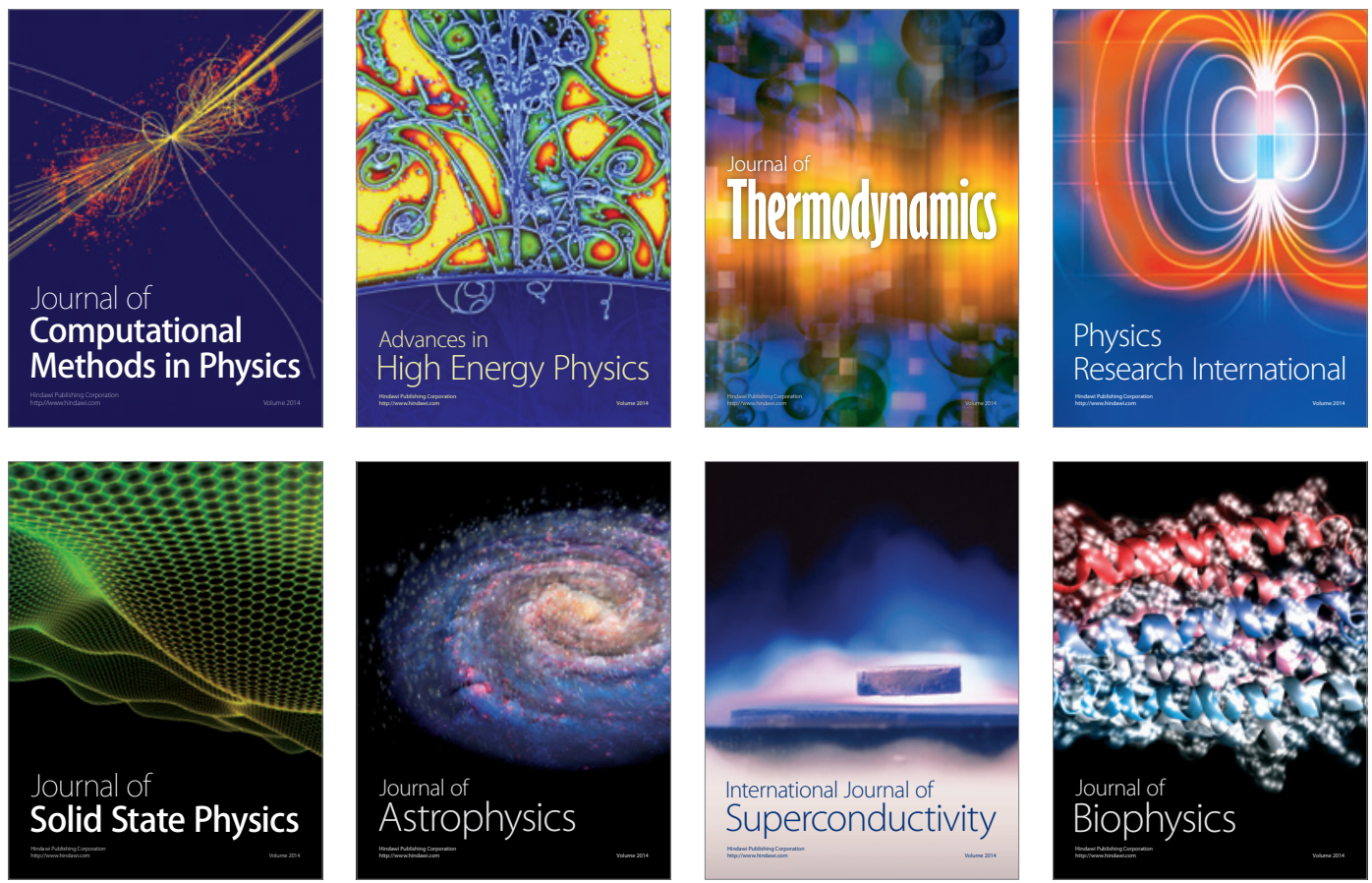
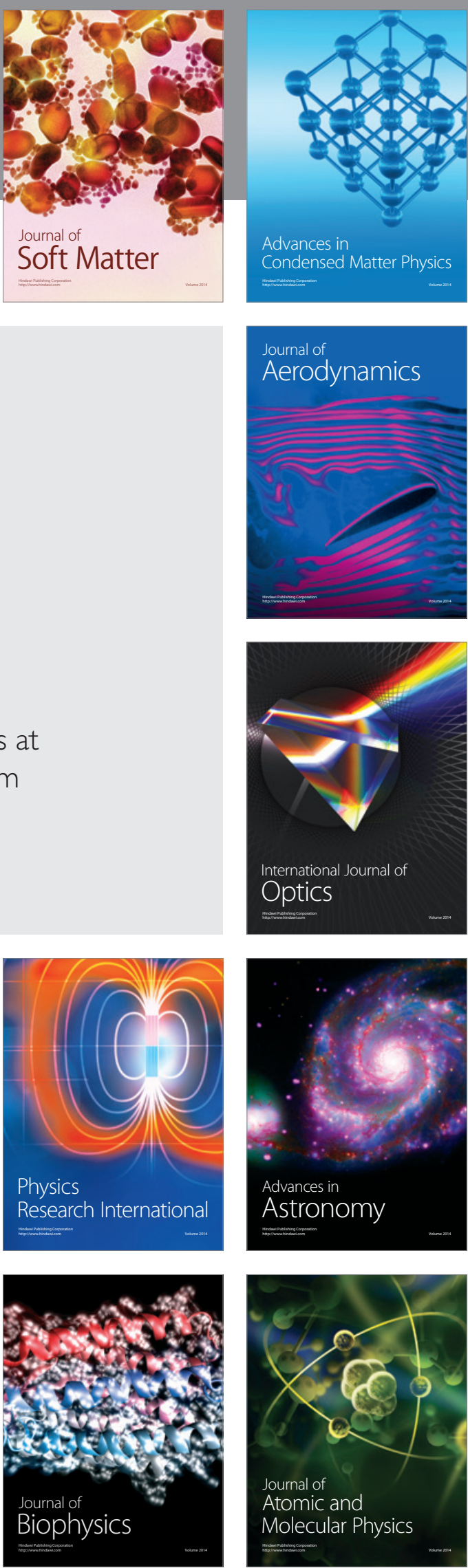